\title{
QUANTUM EFFECTS AND CRITICAL BEHAVIOR IN LOW-DIMENSIONAL SPIN-1/2 SYSTEMS
}

\author{
G. Kamieniarz, G. Musial, L. Dębski, M. Bieliński \\ Computational Physics Division, Institute of Pliysics, A. Mickiewicz University \\ Umultowska 85, 61-614 Poznań, Poland \\ AND R. DEKEYSER \\ Instituut voor Theoretische Fysica, Katholieke Universiteit Leuven \\ Celestijnenlaan 200D, 3001 Leuven, Belgium \\ The critical coupling and spontancous magnetization curve for the 2D- \\ -Ising model are calculated using the effective-ficld method with correlations. \\ An application of the method to the quantum $S=\frac{1}{2}$ 1D-Heisenberg model \\ is presented and reliable low-tempcrature estimates of the specific heat are \\ evaluated.
}

PACS numbers: 64.60.-i, 75.10.11k, 75.10.Jm, 75.40.Mg

We develop the effective-field method with correlations proposed in [1]. For a classical system with short-range interactions the lattice can always be divided into a finite cluster $\Omega$, its boundary $\partial \Omega$, and $\bar{\Omega}$, the complement of $\Omega \bigcup \partial \Omega$, in such a way that its IIamiltonian can be written as $-\beta \mathcal{H}=H_{0}(\Omega, \partial \Omega)+H^{\prime}(\partial \Omega)$, where the degrees of freedom are denoted by the variables $\sigma \in \Omega$ and $\tau \in \partial \Omega$ and the effective IIamiltonian $I^{\prime}(\tau)[1,2]$ can be expanded into the form

$$
H^{\prime}(\tau)=\sum_{i} a_{i} \tau_{i}+\sum_{i \neq j} b_{i j} \tau_{i} \tau_{j}+\ldots
$$

with some self-consistent coefficients $a_{i}, b_{i j}$. First, we consider here the Ising $\Omega$ clusters with $L^{2}$ sites on the square lattice and we calculate the critical coupling and magnetization per spin $\langle\sigma\rangle$ with zero external magnetic field. The estimates of the critical coupling $K_{\mathrm{c}}=J / k_{\mathrm{B}} T_{\mathrm{c}}$ are given in Table, where $L$ denotes the size of the $\Omega$ cluster whereas $n$ is the number of $b$ coefficients taken into account in $H^{\prime}(\tau)$. The exact result is $K_{\mathrm{c}}=\frac{1}{2} \ln (\sqrt{2}+1) \approx 0.4407$. In the second column the MFA results are displayed. The self-consistent calculations with 0, 1,2 and 3 parameters in Eq. (1) for the pair correlations of $\tau$ are performed and the estimates of $K_{\mathrm{c}}$ are shown in the next columns of Table.

The magnetization as a function of $K^{-1}=k_{\mathrm{B}} T / J$ is shown in Fig. 1. The lower thick solid curve is the exact result of Yang [3] while the upper one represents the standard MFA. The sparse dotted curve is calculated for $L=1$ where there 


\section{TABLE}

The estimates of the critical coupling $K_{\mathrm{c}}$ for the 2D-Ising model.

\begin{tabular}{c|c|c|c|c|c}
\hline \hline$L$ & MFA & $n=0$ & $n=1$ & $n=2$ & $n=3$ \\
\hline 1 & 0.250 & 0.346 & & & \\
2 & 0.286 & 0.353 & 0.376 & 0.412 & \\
3 & 0.308 & 0.368 & 0.392 & 0.414 & 0.417 \\
4 & 0.323 & 0.378 & 0.400 & 0.416 & 0.420
\end{tabular}

$\langle\sigma\rangle$

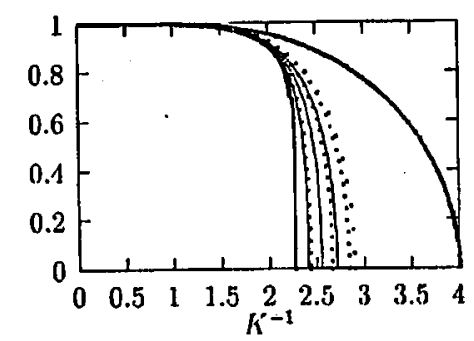

Fig. 1. The magnetization as a function of $k_{\mathrm{B}} T / J$ for $2 \mathrm{D}$-Ising model.

is only one spin $\sigma$ and no correlations are possible to include. The magnetization for $L=2$ is represented by the dense dotted curves and for $L=3$ by the thin solid curves. The upper curves for every $L$ are calculated when no correlations are included in $H^{\prime}(\tau)$, the middle ones when the nearest neighbor correlations are included in $H^{\prime}(\tau)$ and the lower ones when the nearest and the next nearest neighbor correlations are included in $H^{\prime}(\tau)$. Apart from the critical exponent $\beta$ (which retains its classical value), our curves approach the exact magnetization curve in the entire temperature region, even for small clusters.

For quantum-mechanical models the factorization of the Boltzmann factor is not applicable and we should allow the dependence of $H^{\prime}$ on the variables $\sigma$ from the interior of the cluster $\Omega$. We follow the method $[1,2]$ based on the assumption that the strongest contributions to $H^{\prime}$ appear at or around the boundary $\partial \Omega$.

The zero-field specific heat calculated for the isotropic Heisenberg model in the entire temperature region is plotted in Fig. 2. We performed direct diagonalization of the matrix representation with free boundary conditions. We carried out the standard finite-segment calculations $(2 \leq N \leq 15)$ as well as we included up to 2 extra effective interactions. We also performed large-scale quantum transfer-matrix calculations (QTM) [4] for $k_{\mathrm{B}} T / J \leq 0.1$, to obtain independent estimates of the specific heat in the region of quantum fluctuations. The variation of the finite-size specific estimates (per site, in units of $k_{\mathrm{B}}$ ) with respect to $1 / N$ and $1 / \mathrm{m}^{2}$ for both methods at $k_{\mathrm{B}} T / J=0.1$ is plotted in Fig. 3. Our new QTM findings for the system size $N \leq 360$ and the Trotter number $m \leq 13$ confirmed our direct diagonalization datá. We obtained good convergence of the finite-size data and consistent extrapolated values down to $k_{\mathrm{B}} T / J=0.04$. We expect that our 


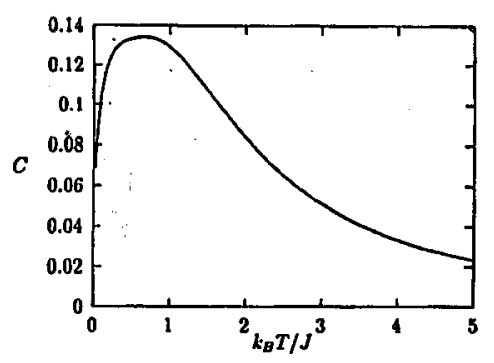

Fig. 2. The zero-field specific heat per site for the 1D-Heisenberg model.

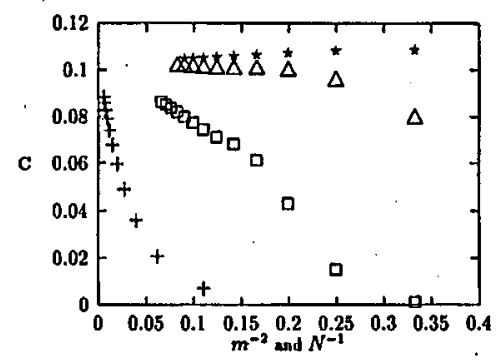

Fig. 3. Variation of the zero-field specific heat per site at $k_{\mathrm{B}} T / J=0.1$ against $1 / N$ for finite-chain diagonalization data $(3 \leq N \leq 15$; the symbols $\square, \Delta, \star$ represent the standard, 1- and 2-parameter approximation, respectively) and against $1 / \mathrm{m}^{2}$ for the transfer-matrix data (+ symbols, $3 \leq m \leq 13$ ).

results will have an impact on simulations of some quasi-one-dimensional systems and also that they will enable calculation of some quantum critical characteristics.

The authors would like to thank the Supercomputing and Networking Center in Poznan for an access to SGI Power Challenge XL, to Cray YMP-EL and to Cray J916. The work was supported in part by the Committee for Scientific Research via the research grant 2 P302 11606.

\section{References}

[1] R. Dekeyser, G. Kamieniarz, J. Magn. Magn. Mater. 104-107, 273 (1992).

[2] G. Kamieniarz, R. Dekeyser, G. Musial, Acta Phys. Pol. A 85, 413 (1994).

[3] C.N. Yang, Phys. Rev. 85, 808 (1952).

[4] T. Delica, H. Leschke, Physica A 168, 736 (1990). 